\title{
The Poset of Mesh Patterns
}

\author{
Jason P. Smith ${ }^{1}$, Henning Ulfarsson ${ }^{2}$
}

\begin{abstract}
We introduce the poset of mesh patterns, which generalises the permutation pattern poset. We fully classify the mesh patterns for which the interval $\left[1^{\emptyset}, m\right]$ is non-pure, where $1^{\emptyset}$ is the unshaded singleton mesh pattern. We present some results on the Möbius function of the poset, and show that $\mu\left(1^{\emptyset}, m\right)$ is almost always zero. Finally, we introduce a class of disconnected and non-shellable intervals by generalising the direct product operation from permutations to mesh patterns.
\end{abstract}

\section{Introduction}

Mesh patterns were first introduced by Brändén and Claesson in [BC11] as a generalisation of permutation patterns, and have been studied extensively in recent years, see e.g., [CTU15, JKR15]. A mesh pattern consists of a pair $(\pi, P)$, where $\pi$ is a permutation and $P$ is a set of coordinates in a square grid. For example, $(312,\{(0,0),(1,2)\})$ is a mesh pattern, which we depict by

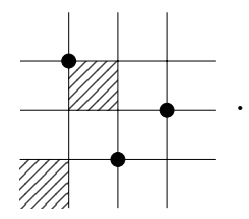

A natural definition of when one mesh pattern occurs in another mesh pattern was given in [TU18], which we present in Section 2. This allows us to generalise the classical permutation poset to a poset of mesh patterns, where $(\sigma, S) \leq(\pi, P)$ if there is an occurrence of $(\sigma, S)$ in $(\pi, P)$. The permutation poset has received a lot of attention in recent years, but

\footnotetext{
${ }^{1}$ This research was supported by the EPSRC Grant EP/M027147/1

${ }^{2}$ Research partially supported by grant 141761-051 from the Icelandic Research Fund
} 
due to its complicated structure a full understanding of it has proven elusive, see [MS15, Smi17]. The poset of mesh patterns, which we define here, contains the poset of permutations as an induced subposet. Therefore, investigating the poset of mesh patterns may lead to a better understanding of the poset of permutations. Moreover, studying this poset may help to answer some of the open questions on mesh patterns.

In Section 2 we introduce the poset of mesh patterns and related definitions, including a brief overview of poset topology. In Section 3 we prove some results on the Möbius function of this poset. In Section 4 we give a characterisation of the non-pure (or non-ranked) intervals of the poset. In Section 5 we give some results on the topology of the poset.

\section{The Poset of Mesh Patterns}

To define a mesh pattern we begin with a permutation $\pi=\pi_{1} \pi_{2} \ldots \pi_{n}$. We can plot $\pi$ on an $n \times n$ grid, where we place a dot at coordinates $\left(i, \pi_{i}\right)$, for all $1 \leq i \leq n$. A mesh pattern is then obtained by shading some of the boxes of this grid, so a mesh pattern takes the form $p=\left(p_{c l}, p_{s h}\right)$, where $p_{c l}$ is a permutation and $p_{s h}$ is a set of coordinates recording the shaded boxes, which are indexed by their south west corner. For ease of notation we sometimes denote the mesh pattern $\left(p_{c l}, p_{s h}\right)$ as $p_{c l}^{p_{s h}}$. We let $\left|p_{c l}\right|$ represent the length of $p_{c l}$ and $\left|p_{s h}\right|$ the size of $p_{s h}$, and define the length of $p$ as $\left|p_{c l}\right|$, which we denote $|p|$. For example, the mesh pattern $(132,\{(0,0),(0,1),(2,2)\})$, or equivalently $132^{(0,0),(0,1),(2,2)}$, has the form:

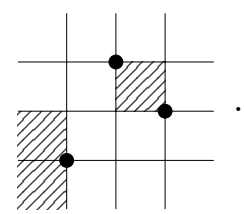

To define when a mesh pattern occurs within another mesh pattern, we first need to recall two other well-known definitions of occurrence. A permutation $\sigma$ occurs in a permutation $\pi$ if there is a subsequence, $\eta$, of $\pi$ whose letters appear in the same relative order of size as the letters of $\sigma$. The subsequence $\eta$ is called an occurrence of $\sigma$ in $\pi$. If no such occurrence exists we say that $\pi$ avoids $\sigma$.

Consider a mesh pattern $(\sigma, S)$ and an occurrence $\eta$ of $\sigma$ in $\pi$, in the classical permutation pattern sense. Each box $(i, j)$ of $S$ corresponds to an area $R_{\eta}(i, j)$ in the plot of $\pi$, which is the rectangle whose corners are the points in $\pi$ which in $\eta$ correspond to the letters $\sigma_{i}, \sigma_{i+1}, j, j+1$ of $\sigma$, 


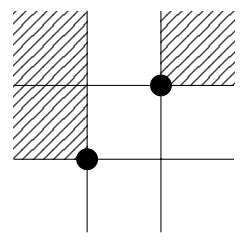

(a)

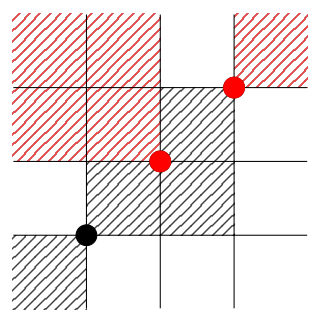

(b)

Figure 2.1: A pair of mesh patterns, with an occurrence of (a) in (b) depicted in red.

and the letters $\sigma_{0}, \sigma_{|\sigma|+1}, 0$ and $|\sigma|+1$ are to the south, north, east and west boundaries, respectively. A point is contained in $R_{\eta}(i, j)$ if it is in the interior of $R_{\eta}(i, j)$, that is, not on the boundary. For example, in Figure 2.1 where $\eta$ is the occurence in red, the area of $R_{\eta}(0,0)$ contains the boxes $\{(0,0),(1,0),(0,1),(1,1)\}$, and it contains exactly one point. We say that $\eta$ is an occurrence of the mesh pattern $(\sigma, S)$ in the permutation $\pi$ if there is no point in $R_{\eta}(i, j)$, for all shaded boxes $(i, j) \in S$.

Using these definitions of occurrences we can recall a concept of mesh pattern containment in another mesh pattern introduced in [TU18]. An example of which is given in Figure 2.1.

Definition 2.1 ([TU18]). An occurrence of a mesh pattern $(\sigma, S)$ in another mesh pattern $(\pi, P)$ is an occurrence $\eta$ of $(\sigma, S)$ in $\pi$, where for any $(i, j) \in S$ every box in $R_{\eta}(i, j)$ is shaded in $(\pi, P)$.

The classical permutation poset $\mathcal{P}$ is defined as the poset of all permutations, with $\sigma \leq_{\mathcal{P}} \pi$ if and only if $\sigma$ occurs in $\pi$. Using Definition 2.1 we can similarly define the mesh pattern poset $\mathcal{M}$ as the poset of all mesh patterns, with $m \leq_{\mathcal{M}} p$ if $m$ occurs in $p$. We drop the subscripts from $\leq$ when it is clear which partial order is being considered. An interval $[\alpha, \beta]$ of a poset is defined as the subposet induced by the set $\{\kappa \mid \alpha \leq \kappa \leq \beta\}$. See Figure 2.2 for an example of an interval of $\mathcal{M}$.

The first result on the mesh pattern poset is that there are infinitely many maximal elements, which shows a significant difference to the permutation poset, where there are no maximal elements.

Lemma 2.2. The poset of mesh pattern contains infinitely many maximal elements, which are the mesh patterns in which all boxes are shaded.

Proof. This follows from the easily proven fact that a fully shaded mesh pattern occurs only in itself, and in no other mesh patterns. 


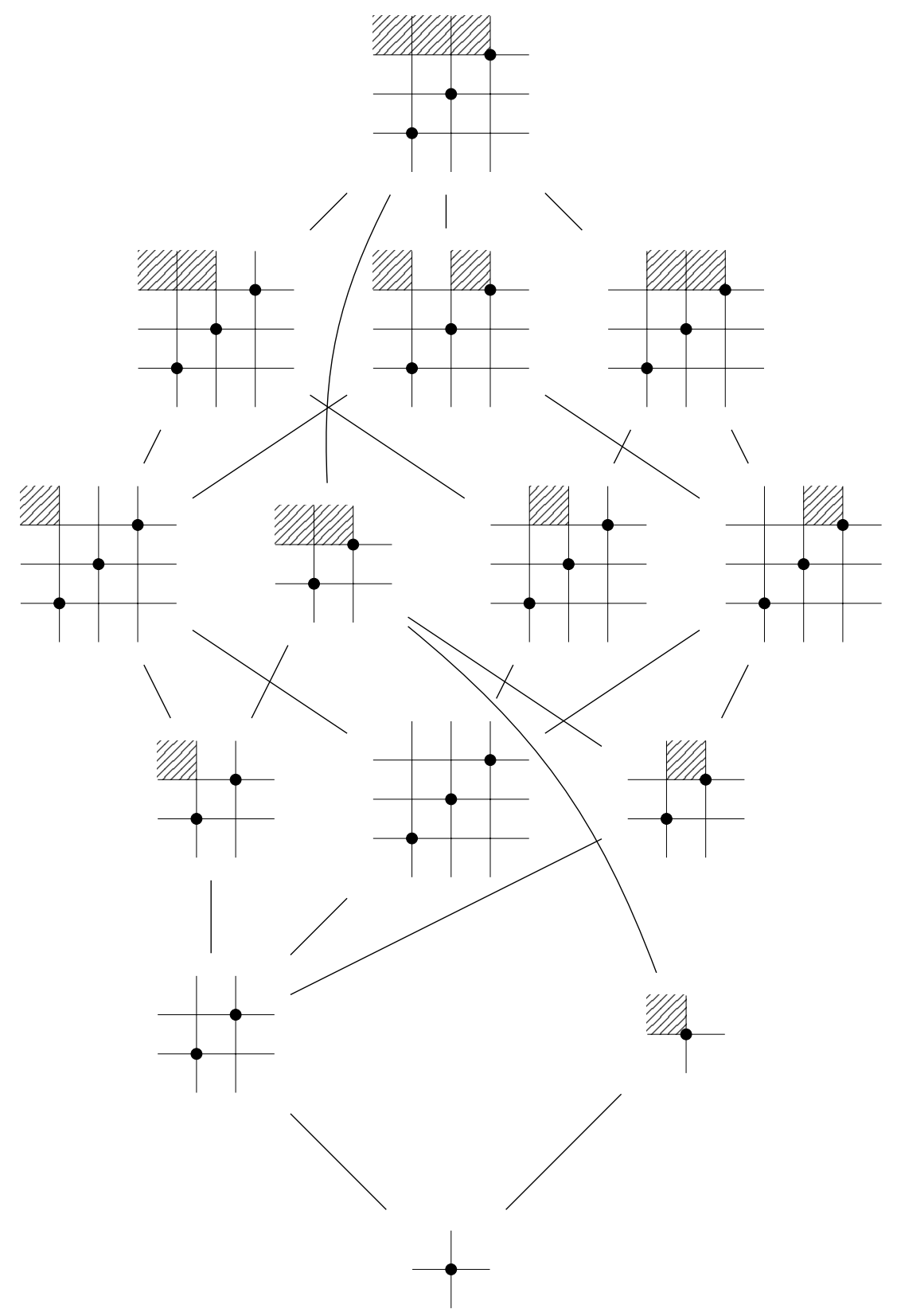

Figure 2.2: The interval $\left[1^{\emptyset}, 123^{(0,3),(1,3),(2,3)}\right]$ of $\mathcal{M}$. 


\subsection{Poset Topology}

In this subsection we briefly introduce some poset topology, and refer the reader to [Wac07] for a comprehensive overview of the topic, including any definitions we omit here.

The Möbius function of an interval $[\alpha, \beta]$ of a poset is defined by: $\mu(a, a)=1$, for all $a, \mu(a, b)=0$ if $a \not \leq b$, and

$$
\mu(a, b)=-\sum_{c \in[a, b)} \mu(a, c) .
$$

See Figure 3.1 for an example. The Möbius function of a poset $P$ is given by $\mu(P)=\mu(\hat{0}, \hat{1})$, where $\hat{0}$ and $\hat{1}$ are unique minimal and maximal elements which we add to $P$.

In a poset we say that $\alpha$ covers $\beta$, denoted $\alpha>\beta$, if $\alpha>\beta$ and there is no $\kappa$ such that $\alpha>\kappa>\beta$. A chain of length $k$ in a poset is a totally ordered subset $c_{1}<c_{2}<\cdots<c_{k+1}$, so the length of a chain is one less than its number of elements. A chain is maximal if $c_{i} \lessdot c_{i+1}$, for all $1 \leq i \leq k$, and $c_{1}$ and $c_{k+1}$ are minimal and maximal elements of the poset, respectively. A poset is pure (also known as ranked) if all maximal chains have the same length. The height of a poset $P$, denoted $h(P)$, is the length of the longest maximal chain. For example, the interval in Figure 2.2 is nonpure because there is one maximal chain of length $3(+\lessdot 4 \lessdot$ maximal chains of length 4 and all other maximal chains have length 5 , so the interval has height 5 .

The interior of an interval $[\alpha, \beta]$ is obtained by removing $\alpha$ and $\beta$, and is denoted $(\alpha, \beta)$. The order complex of an interval $[\alpha, \beta]$, denoted $\Delta(\alpha, \beta)$, is the simplicial complex whose faces are the chains of $(\alpha, \beta)$. When we refer to the topology of an interval we mean the topology of the order complex of the interval. Note that $h(\alpha, \beta)=\operatorname{dim} \Delta(\alpha, \beta)$.

A simplicial complex is shellable if we can order the maximal faces $F_{1}, \ldots, F_{t}$ such that the subcomplex $\left(\cup_{i=1}^{k-1} F_{i}\right) \cap F_{k}$ is pure and $\left(\operatorname{dim} F_{k}-1\right)$ dimensional, for all $k=2, \ldots, t$. So a poset is shellable if there is an ordering of the maximal chains such that the intersection given above is pure and has height $\left(h\left(F_{k}\right)-1\right)$. Being shellable implies other properties on the topology, such as having the homotopy type of a wedge of spheres.

An interval $I$ is disconnected if the interior can be split into two disjoint pairwise incomparable sets, that is, $I=A \cup B$ with $A \cap B=\emptyset$ and for every $a \in A$ and $b \in B$ we have $a \not \leq b$ and $b \not \leq a$. Each interval $I$ can be decomposed into its smallest connected parts, which we call the components of $I$. A component is nontrivial if it contains more than one element and 
we say an interval is strongly disconnected if it has at least two nontrivial components. For example, the interval $\left[1^{\emptyset}, 12^{(0,2),(1,2)}\right]$ in Figure 2.2 is disconnected but not strongly disconnected. Note that if an interval has height less than 3 it can never be strongly disconnected.

It is well known that disconnectivity is a barrier to shellability when an interval is pure, see [MS15]. In the non-pure case the situation is similar, which can be seen in the following result.

Lemma 2.3. If an interval is strongly disconnected, then it is not shellable.

Proof. Consider any ordering of the maximal chains and let $F_{k}$, with $k>1$, be the first chain where every preceding chain belongs to a different component and $F_{k}$ belongs to a nontrivial component. Note that such an $F_{k}$ exists in every ordering because the interval is strongly disconnected, and because $F_{k}$ belongs to a nontrivial component it must have length at least 1 . So $\left(\cup_{i=1}^{k-1} F_{i}\right) \cap F_{k}=\emptyset$, which has height $-1 \neq\left(h\left(F_{k}\right)-1\right)$. Therefore, the ordering is not a shelling.

Since every subinterval of a shellable interval is shellable, [Wac07, Corollary 3.1.9], we obtain the following:

Corollary 2.4. An interval which contains a strongly disconnected subinterval is not shellable.

Finally, we present a useful result known as the Quillen Fiber Lemma [Qui78]. Two simplicial complexes are homotopy equivalent if one can be obtained by deforming the other but not breaking or creating any new "holes", for a formal definition see [Hat02]. A simplicial complex is contractible if it is homotopy equivalent to a point and if two posets are homotopy equivalent their Möbius functions are equal. Given a poset $P$, with $p \in P$ define the upper ideal $P_{\geq p}=\{q \in P \mid q \geq p\}$.

Proposition 2.5. (Quillen Fiber Lemma) Let $\phi: P \rightarrow Q$ be an orderpreserving map between posets such that for any $x \in Q$ the complex $\Delta\left(\phi^{-1}\left(Q_{\geq x}\right)\right)$ is contractible. Then $P$ and $Q$ are homotopy equivalent.

\section{Möbius Function}

In this section we present some results on the Möbius function of the mesh pattern poset. We begin with some simple results on: mesh patterns with the same underlying permutations; the mesh patterns with no points $\epsilon^{\emptyset}$ and $\epsilon^{(0,0)}$; and mesh patterns with no shaded boxes. Throughout the remainder of the paper we assume that $m$ and $p$ are mesh patterns. 
Lemma 3.1. Let $\pi$ be a permutation. For any sets $A \subseteq B$ the interval $\left[\pi^{A}, \pi^{B}\right]$ is isomorphic to the boolean lattice $B_{|B|-|A|}$. Therefore, $\mu\left(\pi^{A}, \pi^{B}\right)=(-1)^{|B|-|A|}$ and $\left[\pi^{A}, \pi^{B}\right]$ is shellable.

Proof. The elements of $\left[\pi^{A}, \pi^{B}\right]$ are exactly the mesh patterns $\pi^{A \cup C}$ where $C \subseteq B \backslash A$, which implies the result.

Lemma 3.2. Consider $A \in\{\emptyset,(0,0)\}$, then:

$$
\mu\left(\epsilon^{A}, p\right)= \begin{cases}1, & \text { if } p=\epsilon^{A} \\ -1, & \text { if } A=\emptyset \&\left|p_{c l}\right|+\left|p_{s h}\right|=1 \\ 0, & \text { otherwise }\end{cases}
$$

Proof. The first two cases are trivial. By the proof of Lemma 2.2 we know that $\epsilon^{(0,0)}$ is not contained in any larger mesh patterns, which implies $\mu\left(\epsilon^{(0,0)}, p\right)=0$, for all $p \neq \epsilon^{(0,0)}$. If $\left|p_{c l}\right|+\left|p_{s h}\right|>1$, then $\left(\epsilon^{\emptyset}, p\right)$ contains a unique minimal element $1^{\emptyset}$, so $\mu\left(\epsilon^{\emptyset}, p\right)=0$.

Lemma 3.3. The interval $\left[\sigma^{\emptyset}, \pi^{\emptyset}\right]$ is isomorphic to $[\sigma, \pi]$ in $\mathcal{P}$, so

$$
\mu_{\mathcal{M}}\left(\sigma^{\emptyset}, \pi^{\emptyset}\right)=\mu_{\mathcal{P}}(\sigma, \pi) .
$$

The Möbius function $\mu(\sigma, \pi)$ of the classical permutation poset is known to be unbounded [SV06]. So we get the following corollary:

Corollary 3.4. The Möbius function $\mu(m, p)$ is unbounded on $\mathcal{M}$.

We can also show that the Möbius function is unbounded if we include shaded boxes. We do this by mapping to the poset $\mathcal{W}$ of words with subword order, that is, the poset made up of all words and $u \leq w$ if there is a subword of $w$ that equals $u$. The map we introduce is analogous to the map in [Smi16, Section 2], which maps certain intervals of the permutation poset to intervals of $\mathcal{W}$. A descent in a permutation $\pi=\pi_{1} \pi_{2} \ldots \pi_{n}$ is a pair of letters $\pi_{i}, \pi_{i+1}$ with $\pi_{i}>\pi_{i+1}$. We call $\pi_{i+1}$ the descent bottom. An adjacency tail is a letter $\pi_{i}$ with $\pi_{i}=\pi_{i-1} \pm 1$. Let $\operatorname{adj}(\pi)$ be the number of adjacency tails in $\pi$. Consider the set $\Gamma$ of mesh patterns where the permutation has exactly one descent, the descent bottom is 1 and we shade everything south west of 1 . For example, the mesh pattern $2314^{(0,0),(1,0),(2,0)}$ :

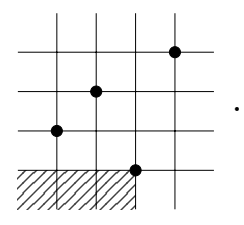


Lemma 3.5. Consider a mesh pattern $m \in \Gamma$, then $\left[21^{(0,0),(1,0)}, m\right]$ is shellable and $\mu\left(21^{(0,0),(1,0)}, m\right)= \begin{cases}(-1)^{|m|}\left\lfloor\frac{|m|}{2}\right\rfloor, & \text { if } \operatorname{adj}\left(m_{c l}\right)=0 \\ (-1)^{|m|}, & \text { if } \operatorname{adj}\left(m_{c l}\right)=1 \text { \& tail before descent } . \\ 0, & \text { otherwise }\end{cases}$

Proof. First note that every mesh pattern in $\left[21^{(0,0),(1,0)}, m\right]$ is also in $\Gamma$. We define a map $f$ from $\Gamma$ to binary words in the following way. Let $b(x)$ be the set of letters that appear before 1 in $x \in \Gamma$. Set $\hat{f}(x)$ as the word where the $i$ th letter is 0 if it is in $b(x)$ and 1 otherwise, and let $f(x)$ equal $\hat{f}(x)$ with the first letter removed. So $f(\Gamma)$ is the set of binary words with at least one 0 . The inverse of this map is obtained by the following procedure: 1 ) take a binary word $w \in f(\Gamma)$ and prepend a $1 ; 2)$ put the positions that are 0's in increasing order followed by the positions that are 1 in increasing order; and 4) shade everything southwest of 1 . So $f$ is a bijection.

It is straightforward to check that $f$ is order preserving. So the interval $\left[21^{(0,0),(1,0)}, m\right]$ is isomorphic to $[0, f(m)]$ in $\mathcal{W}$. It was shown in [Bjö90] that intervals of $\mathcal{W}$ are shellable, which proves the shellability part. It was also shown that the Möbius function equals the number of normal occurrences with the sign given by the height, where an occurrence is normal if in any consecutive sequence of equal elements every non-initial letter is part of the occurrence. So for an occurrence of 0 in $f(m)$ to be normal there can be no 1 directly preceded by a 1 and at most one 0 directly preceded by a 0 . If such a 0 exists it must be the occurrence, otherwise any 0 can be the occurrence. In our bijection a non-initial letter of such a sequence maps to an adjacency tail. Combining this with the fact that if there are no adjacency tails, then the letters before the descent must be all the even letters of which there are $\left\lfloor\frac{|m|}{2}\right\rfloor$, completes the proof.

The Möbius function on $\mathcal{P}$ often takes larger values than on $\mathcal{M}$, but it is not always true that $\mu_{\mathcal{M}}(m, p) \leq \mu_{\mathcal{P}}\left(m_{c l}, p_{c l}\right)$. A simple counterexample is the interval

$$
\left[1^{(0,1)}, 123^{(0,2),(0,3),(1,2),(1,3)}\right]
$$

which has Möbius function 1 , however $\mu_{\mathcal{P}}(1,123)=0$, see Figure 3.1.

If we consider intervals where the bottom mesh pattern has no shadings, then we get the following result:

Lemma 3.6. Consider an interval $\left[s^{\emptyset}, p\right]$ in $\mathcal{M}$ with $p_{\text {sh }} \neq \emptyset$. If $s^{B} \notin\left(s^{\emptyset}, p\right)$ for any set $B$, then $\mu\left(s^{\emptyset}, p\right)=0$. 

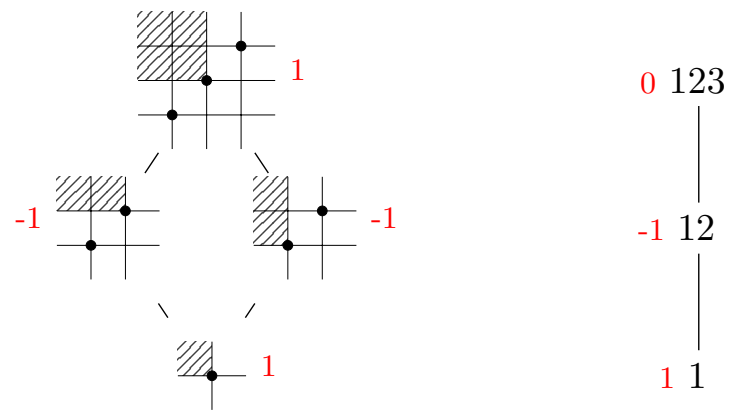

Figure 3.1: The interval $\left[1^{(0,1)}, 123^{(0,2),(0,3),(1,2),(1,3)}\right]$ (left) in $\mathcal{M}$ and $[1,123]$ (right) in $\mathcal{P}$, with the Möbius function in red.

Proof. Consider the map $f:\left(s^{\emptyset}, p\right) \rightarrow A: x \mapsto x_{c l}^{\emptyset}$, that is, $f$ removes all shadings from $x$. We can see that $A=\left(s^{\emptyset}, p_{c l}^{\emptyset}\right]$, so $A$ is contractible, because it has the unique maximal element $p_{c l}^{\emptyset}$, hence $\mu(A)=0$. Moreover, $f^{-1}\left(A_{\geq y}\right)=[y, p)$, for all $y \in A$, which is contractible. Therefore, $\left(s^{\emptyset}, p\right)$ is homotopy equivalent to $A$ by the Quillen Fiber Lemma (Proposition 2.5), which implies $\mu\left(s^{\emptyset}, p\right)=0$.

Example 3.7. Consider the subinterval $\left[1^{\emptyset}, 12^{(0,2)}\right]$ in Figure 2.2, applying Lemma 3.6 implies $\mu\left(1^{\emptyset}, 12^{(0,2)}\right)=0$. However, we cannot apply Lemma 3.6 to $\left[1^{\emptyset}, 12^{(0,2),(1,2)}\right]$ because it contains the element $1^{(0,1)}$.

We can combine Lemma 3.6 with the following result to see that the Möbius function is almost always zero on the interval $\left[1^{\emptyset}, p\right]$.

Lemma 3.8. As $n$ tends to infinity the proportion of mesh patterns of length $n$ that contain any of $\left\{1^{(0,0)}, 1^{(1,0)}, 1^{(0,1)}, 1^{(1,1)}\right\}$ approaches 0 .

Proof. Let $P(n, i)$ be the probability that the letter $i$ is an occurrence of $1^{(0,0)}$ in a length $n$ mesh pattern, and let $P(n)$ be the probability that a length $n$ mesh pattern contains $1^{(0,0)}$.

The probability $P(n, i)$ can be bounded above by first considering the index $k$ of $i$, each having probability $\frac{1}{n}$, and then requiring that all boxes south west of $i$ are filled, of which there are $i k$. This provides an upper bound, because it is possible that there is a point south west of $i$, which 
would imply $i$ is not an occurrence of $1^{(0,0)}$. We can formulate this as:

$$
\begin{aligned}
P(n, i) & \leq \sum_{k=1}^{n} \frac{1}{n}\left(\frac{1}{2^{i}}\right)^{k}=\frac{1}{n}\left(\frac{1-2^{-i(n+1)}}{1-2^{-i}}-1\right) \\
& =\frac{1}{n}\left(\frac{2^{-i}-2^{-i(n+1)}}{1-2^{-i}}\right)=\frac{1}{n 2^{i}}\left(\frac{1-2^{-i n}}{1-2^{-i}}\right) \leq \frac{2}{n 2^{i}}
\end{aligned}
$$

To compute the probability $P(n)$ we can sum over all the $P(n, i)$. Note again this is an over estimate because if a mesh pattern contains multiple occurrences of $1^{(0,0)}$ it counts that mesh pattern more than once.

$$
P(n) \leq \sum_{i=1}^{n} P(n, i) \leq \sum_{i=1}^{n} \frac{2}{n 2^{i}}=\frac{2}{n}\left(\frac{1-\left(\frac{1}{2}\right)^{n+1}}{1-\frac{1}{2}}-1\right) \leq \frac{2}{n}
$$

Repeating this calculation for the other three shadings of 1 implies that the probability of containing any of the forbidden mesh patterns is bounded by $\frac{8}{n}$ which tends to zero as $n$ tends to infinity.

Because of the previous lemma we obtain:

Corollary 3.9. As $n$ tends to infinity the proportion of mesh patterns $p$ of length $n$ such that $\mu\left(1^{\emptyset}, p\right)=0$ approaches 1 .

In the classical case it is true that given a permutation $\sigma$ the probability a permutation of length $n$ contains $\sigma$ tends to 1 as $n$ tends to infinity, this follows from the Marcus-Tardos Theorem [MT04]. By the above result we can see the same is not true in the mesh pattern case. In fact we conjecture the opposite is true:

Conjecture 3.10. Given a mesh pattern $m$, with at least one shaded box, the probability that a random mesh pattern of length $n$ contains $m$ tends to 0 as $n$ tends to infinity.

\section{Purity}

Recall that a poset is pure (also known as ranked) if all the maximal chains have the same length, and as we can see from Figure 2.2, intervals of the mesh pattern poset can be non-pure. In this section we classify which intervals $\left[1^{\emptyset}, m\right]$ are non-pure. First we consider the length of the longest maximal chain in any interval $\left[1^{\emptyset}, m\right]$, that is, the height of $\left[1^{\emptyset}, m\right]$.

Lemma 4.1. For a mesh pattern $m$, we have $h\left(1^{\emptyset}, m\right)=\left|m_{c l}\right|+\left|m_{s h}\right|$. 

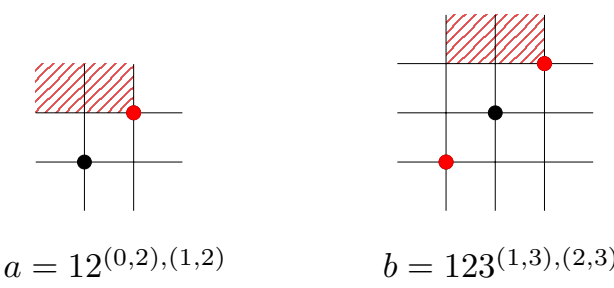

$$
a=12^{(0,2),(1,2) \quad b=123^{(1,3),(2,3)}}
$$

Figure 4.1: Two mesh patterns with a point $x$ in black whose deletion merges shadings and the occurrences $\eta_{a}^{x}$ and $\eta_{b}^{x}$ in red. By Lemma 4.2 the edge $a_{x}^{-} \lessdot a$ is impure, but $b_{x}^{-}<b$ is not, because there is a second occurrence of $b_{x}^{-}$in $b$, using points 23 , that does not use all the shaded boxes in $b$.

Proof. We can create a chain from $m$ to $1^{\emptyset}$ by deshading all boxes, in any order, and then deleting all but one point, in any order. The length of this chain is $\left|m_{c l}\right|+\left|m_{s h}\right|$. Moreover, we cannot create a longer chain because at every step of a chain we must deshade a box or delete a point.

Therefore, we define the height of a mesh pattern as $h(m)=\left|m_{c l}\right|+\left|m_{s h}\right|$ and we say an edge $m \lessdot p$ is impure if $h(p)-h(m)>1$. Next we give a classification of impure edges.

Let $m_{x}^{-}$be the mesh pattern obtained by deleting the point $x$ in $m$ and let $\eta_{m}^{x}$ be the occurrence of $m_{x}^{-}$in $m$ that does not use the point $x$. An occurrence $\eta$ of $m$ in $p$ uses the shaded box $(a, b) \in p_{s h}$ if $(a, b) \in R_{\eta}(i, j)$ for some shaded box $(i, j) \in m_{s h}$. We say that deleting a point $x$ merges shadings if there is a shaded box in $m_{x}^{-}$that corresponds to more than one shaded box in $\eta_{m}^{x}$, see Figure 4.1. Also note that if deleting a point merges an unshaded box with a shaded box then the resulting box will be unshaded, so we say deleting that point removes shadings. Note that is is possible that deleting a point both merges and removes shadings. We define a block of shaded boxes to be a consecutive sequence of shaded boxes in the same row or column.

Lemma 4.2. Two mesh patterns $m<p$ form an impure edge if and only if all occurrences of $m$ in $p$ use all the shaded boxes of $p$ and are obtained by deleting a point that merges shadings.

Proof. First we show the backwards direction. Because $m$ is obtained by deleting a point that merges shadings, $m$ must have one less point and at least one less shaded box so $h(p)-h(m) \geq 2$. So it suffices to show that there is no $z$ such that $m<z<p$. Suppose such a $z$ exists, then if $z$ is obtained by deshading a box in $p$ it can no longer contain $m$ because all occurrences 
of $m$ in $p$ use all the shaded boxes of $p$. If $z$ is obtained by deleting a point and $m<z$, then $m_{c l}=z_{c l}$. Therefore, we can deshade some boxes of $z$ to get $m$, which implies there is an occurrence of $m$ in $p$ that does not use all the shaded boxes of $p$.

Now consider the forward direction. Suppose $m \lessdot p$ is impure, so $h(p)-$ $h(m) \geq 2$. Therefore, $m$ is obtained by deleting a single point which merges shadings, but does not delete shadings because any other combination of deleting points and deshading can be done in successive steps. Furthermore, this must be true for any point that can be deleted to get $m$, that is, for all occurrences of $m$ in $p$. Moreover, if there is an occurrence that does not use all the shaded boxes of $p$, we can deshade the box it doesn't use and get an element that lies between $m$ and $p$.

Lemma 4.3. Suppose that deleting positions $i$ or $j$ from $m$ gives the same mesh pattern $p$, and their deletion does not remove shaded boxes. Then deleting any position $k$ from $m$, with $i \leq k \leq j$, also gives $p$ and does not remove shaded boxes. Moreover, deleting $i$ and $k$ gives the same mesh pattern as deleting $k$ and $j$.

Proof. Let $m_{x}$ be the value of the point in position $x$ of $m$, and assume $m_{i}<$ $m_{j}$, the case for $m_{i}>m_{j}$ is analogous. It is well known that deleting two letters from a permutation gives the same subpermutation if and only if they are part of the same adjacency, that is, sequence of consecutively valued letters in consecutive positions, see [Smi17]. So if deleting $i$ and $j$ gives the same mesh pattern they must be part of the same adjacency, so $k$ must also be part of the same adjacency, hence deleting $k$ gives the same underlying permutation, and $m_{i}<m_{k}<m_{j}$. It remains to show that deleting $k$ results in the same shaded boxes as deleting $i$ or $j$. We prove the following claim:

If deleting positions $i$ or $j$ from $m$ gives the same mesh pattern $p$, and their deletion does not remove shaded boxes, then for every column $c$ (resp. row $r$ ) the boxes $\left(c, m_{x}\right)$ (resp. $\left.(x, r)\right)$ are either all shaded or all unshaded, for $i-1 \leq x \leq j$.

Note that the implication of the claim is equivalent to saying that every row $\pi_{i}-1 \leq r \leq \pi_{j}$ has exactly the same shaded boxes, and every column $i-$ $1 \leq c \leq j$ has exactly the same shaded boxes.

First we consider a column $c<i-1$. If deleting $i$ or $j$ removes a shaded boxes then this is true for both $i$ and $j$, otherwise column $a$ in the resulting mesh patterns would have differing number of shaded boxes. If the deletion of $\mathrm{i}$ or $\mathrm{j}$ does not merge boxes, then there cannot be a shaded 
box $(c, x)$ with $m_{i}<x<m_{j}-1$. To see this suppose $(c, x)$ is the lowest such shaded box, then deleting $i$ causes $(c, x-1)$ to be shaded, but $(c, x-1)$ is unshaded after deleting $j$. A similar argument shows there cannot be an unshaded box $(c, x)$ with $m_{i}<x<m_{j}-1$ if deleting $i$ and $j$ merge shadings. Analogous arguments prove the same is true for any column $c>j$, row $r<m_{i}-1$ or row $r>m_{j}$.

It remains to consider the rectangle given by the boxes $(c, r)$ with $i-1 \leq$ $c \leq j$ and $\pi_{i}-1 \leq r \leq \pi_{j}$, for which the claim is equivalent to saying this rectangle is either all shaded or all unshaded. Note that deleting $i$ causes any box in this rectangle to be shifted down and left. So if $(a, b)$ is shaded, then $(a-1, b-1)$ and $(a+1, b+1)$ are also shaded, thus all boxes in that diagonal line within the rectangle are shaded. The north eastern most pair $(x-1, y-1)$ and $(x, y)$ of this diagonal are either in columns $j-1$ and $j$ or rows $m_{j}-1$ and $m_{j}$, and are shaded. Therefore, the boxes $(x, y-1)$ and $(x-1, y)$ are also shaded since deleting $j$ does not remove shaded boxes. So the diagonals containing these two boxes, which are the ones above and below the original diagonal, are also fully shaded. Thus by induction every diagonal is fully shaded, so the whole rectangle is fully shaded. This completes the proof of the claim.

So deleting any point $i \leq k \leq j$ cannot remove shaded boxes, as that would imply there is a row or column with neighbouring shaded and unshaded boxes between points $i$ and $j$. Moreover, deleting $k$ is equivalent to merging two identical rows and two identical columns, which is the same as removing one row and one column, and as all the rows and columns are the same we end up with the same shadings irrelevant of which is removed. Furthermore, deleting any pair of points is equivalent to deleting any two rows and two columns, which results in the same mesh pattern, which proves the final part of the lemma.

Lemma 4.4. If $[m, p]$ contains an impure edge, then it contains an impure edge $a \lessdot b$ where $b_{c l}=p_{c l}$.

Proof. First note that inserting a point into a mesh pattern can only create more shaded boxes by splitting previously shaded boxes ${ }^{3}$. So inserting a letter into an occurrence that uses all the shaded boxes creates a new occurrence, of a longer pattern, with the same property.

\footnotetext{
${ }^{3} \mathrm{~A}$ point inserted in box $(i, j)$ splits every box in row $i$ and column $j$ into two boxes, except the box $(i, j)$ itself, which splits into four boxes.
} 
Suppose $x \lessdot y$ is an impure edge in $[m, p]$, and let $\eta$ be an occurrence of $x$ in $y$. Let $\hat{\eta}$ be an occurrence of $\hat{x}$ in $\hat{y}$, where $\hat{y}$ is obtained by inserting some letter $t$ into $y$, then $\hat{\eta}$ is obtained by inserting $t$ into $\eta$, and $\hat{x}$ is uniquely determined by $\hat{\eta}$. Given any occurrence $\psi$ of $x$ in $y$ (resp. $\hat{x}$ in $\hat{y}$ ) let $m(\psi)$ be the letter deleted from $y$ (resp. $\hat{y}$ ) to get $\psi$. We show that every occurrence $\hat{\psi}$ of $\hat{x}$ in $\hat{y}$ uses all the shaded boxes.

If $\hat{\psi}$ contains $t$, we show that removing $t$ from $\hat{\psi}$ gives an occurrence $\psi$ of $x$ in $y$. Note that removing $t$ from $\hat{y}$ gives $y$ by construction. If $m(\hat{\psi})$ and $m(\hat{\eta})$ are both before $t$ or both after $t$, then $t$ appears as the same letter of $\hat{x}$ in both occurrences, and removing $t$ from $\hat{\eta}$ gives an occurrence of $x$, so the same must be true for $\hat{\psi}$. If $t$ is between $m(\hat{\psi})$ and $m(\hat{\eta})$, then note that deleting either $m(\hat{\psi})$ or $m(\hat{\eta})$ gives $\hat{x}$, since both $\hat{\psi}$ and $\hat{\eta}$ are occurrences of $\hat{x}$ in $\hat{y}$. So by Lemma 4.3 deleting $m(\hat{\psi})$ and $t$ gives the same mesh pattern as deleting $m(\hat{\eta})$ and $t$, which is $x$. So $\psi$ is an occurrence of $x$ in $y$ that uses all the shaded boxes, hence $\hat{\psi}$ also uses all the shaded boxes by the first paragraph. If $\hat{\psi}$ does not contain $t$, then as $|\hat{x}|=|\hat{y}-1|$ we know that $t$ is the only letter not contained in $\hat{\psi}$, which implies that deleting $t$ from $\hat{y}$ gives $\hat{x}$. Moreover, by the definition of $\hat{y}$ we know that deleting $t$ from $\hat{y}$ gives $y$, so $\hat{x}=y$. Again, by the first paragraph we know that $\hat{\psi}$ must use all the shaded boxes, because $\hat{y}$ is constructed by inserting $t$ into $y$.

Now we show that deleting $m(\hat{\psi})$ merges shaded boxes. Deleting $m(\hat{\psi})$ or $m(\hat{\eta})$ gives $\hat{x}$, so the resulting number of shaded boxes must be the same after deleting either, and we know that deleting $m(\hat{\eta})$ merges shaded boxes by construction, so the number of shaded boxes must decrease. Moreover, as $\hat{\psi}$ uses all the shaded boxes the only way to decrease the number of shaded boxes is to merge them.

All occurrences of $\hat{x}$ in $\hat{y}$ satisfy the conditions of Lemma 4.2, hence $\hat{x} \lessdot \hat{y}$ is an impure edge. The result then follows by induction, as we can sequentially insert points into $y$ to create a mesh pattern $b$ with $b_{c l}=p_{c l}$, which will cover an element $a$ such that $a \lessdot b$ is impure.

Proposition 4.5. The interval $\left[1^{\emptyset}, m\right]$ is non-pure if and only if there exists a point $x$ in $m$ whose deletion merges shadings and there is no other occurrence of $m_{x}^{-}$in $m$ which uses a proper subset of the shadings used by $\eta_{m}^{x}$.

Proof. First we show the backwards direction. Let $t$ be the mesh pattern obtained by inserting $x$ back into $m_{x}^{-}$, and $\phi$ the corresponding occurrence of $m_{x}^{-}$in $t$. Note that $\phi$ and $\eta_{m}^{x}$ use the same set of shadings, but $m$ may have more shaded boxes than $t$. We know that deleting $x$ from $m$ merges shadings, which implies that $\phi$ uses all the shaded boxes of $t$. Also note 


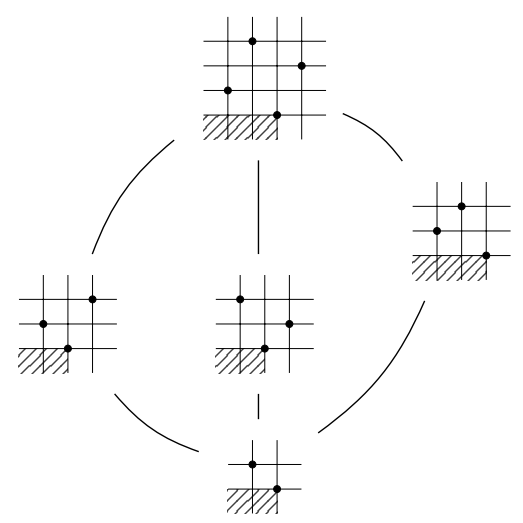

Figure 4.2: The interval $\left[21^{(0,0),(1,0)}, 2413^{(0,0),(1,0),(2,0)}\right]$, which is pure but contains both pure and impure edges.

that any other occurrences of $m_{x}^{-}$in $t$ must also use all of the shaded boxes because there is no occurrence of $m_{x}^{-}$in $m$ which uses a proper subset of the shadings used by $\phi$. Therefore, by Lemma 4.2 we get that $m_{x}^{-} \lessdot t$ is an impure edge.

To see the other direction suppose there is an impure edge in $\left[1^{\emptyset}, m\right]$. By Lemma 4.4 there is an impure edge $a \lessdot b$ where $b_{c l}=m_{c l}$. By Lemma 4.2 all occurrences of $a$ in $b$ use all shaded boxes of $b$ and are obtained by deleting a point that merges shadings. Moreover, if deleting a point merges shadings in $b$, then its deletion merges shadings in $m$, which implies the result.

Corollary 4.6. There is an impure edge in the interval $[m, p]$ if and only if there exists a point $x$ in $p$ whose deletion merges shadings and there is no other occurrence of $p_{x}^{-}$in $p$ with a subset of shadings of $\eta_{p}^{x}$, and $p_{x}^{-} \geq m$.

Note that containing an impure edge in $[m, p]$ does not necessarily imply that $[m, p]$ is non-pure. For example, if $[m, p]$ contains only one edge and that edge is impure, then $[m, p]$ is still pure. Moreover, it is possible to have a pure poset that contains impure and pure edges, see Figure 4.2.

\section{Topology}

A full classification of shellable intervals has not been obtained for the classical permutation poset, so finding such a classification for the mesh pattern poset would be equally difficult, if not more so. However, in [MS15] all disconnected intervals of the permutation poset are described, and containing a disconnected subinterval implies a pure interval is not shellable. So this 
gives a large class of non-shellable intervals, in fact it is shown that almost all intervals are not shellable. We showed in Lemma 2.3 that containing a strongly disconnected interval implies an interval is not shellable. So in this section we consider when an interval is strongly disconnected. Firstly we look at the relationship between connectivity in $\mathcal{P}$ and $\mathcal{M}$.

The connectivity of the interval $\left[m_{c l}, p_{c l}\right]$ in $\mathcal{P}$ does not necessarily imply the same property for $[m, p]$ in $\mathcal{M}$. For example, the interval $[123,456123]$ is disconnected in $\mathcal{P}$ but the interval

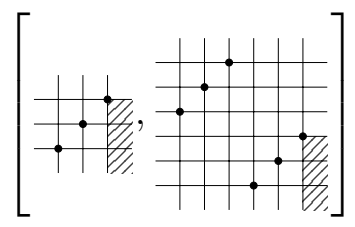

is a chain in $\mathcal{M}$, so is connected. Furthermore, the interval [321, 521643] is connected in $\mathcal{P}$ but the interval

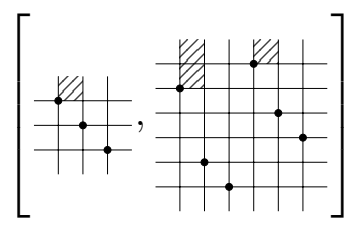

is strongly disconnected in $\mathcal{M}$. Therefore, if $\left[m_{c l}, p_{c l}\right]$ is (non-)shellable in $\mathcal{P}$, then it is not true that $[m, p]$ has the same property in $\mathcal{M}$. For example, $[123,456123]$ is not shellable but $(5.1)$ is shellable, and $[321,521643]$ is shellable but (5.2) is not shellable.

In [MS15] the direct sum operation is used to show that almost all intervals of the permutation poset are not shellable in $\mathcal{P}$. We generalise the direct sum operation to mesh patterns. Given two permutations $\alpha=\alpha_{1} \ldots \alpha_{a}$ and $\beta=\beta_{1} \ldots \beta_{b}$ the direct sum of the two is defined as $\alpha \oplus \beta=\alpha_{1} \ldots \alpha_{a}\left(\beta_{1}+\right.$ $a)\left(\beta_{2}+a\right) \ldots\left(\beta_{b}+a\right)$, that is, we increase the value of each letter of $\beta$ by the length of $\alpha$ and append it to $\alpha$. This can also be thought of in terms of the plots of $\alpha$ and $\beta$ by placing a copy of $\beta$ to the north east of $\alpha$. Similarly we can define the skew-sum $\alpha \ominus \beta$ by prepending $\alpha$ to $\beta$ and increasing the value of each letter of $\alpha$ by the length of $\beta$. We extend these definitions to mesh patterns in the following way:

Definition 5.1. Consider two mesh patterns $s$ and $t$, where the top right corner of $s$ and bottom left corner of $t$ are not shaded. The direct sum $s \oplus t$ has the classical pattern $s_{c l} \oplus t_{c l}$ and shaded boxes $s_{s h} \cup\left\{\left(i+\left|s_{c l}\right|, j+\right.\right.$ $\left.\left.\left|s_{c l}\right|\right) \mid(i, j) \in t_{s h}\right\}$, and also for any shaded boxes $\left(i,\left|s_{c l}\right|\right),\left(\left|s_{c l}\right|, i\right),\left(j,\left|s_{c l}\right|\right)$ 


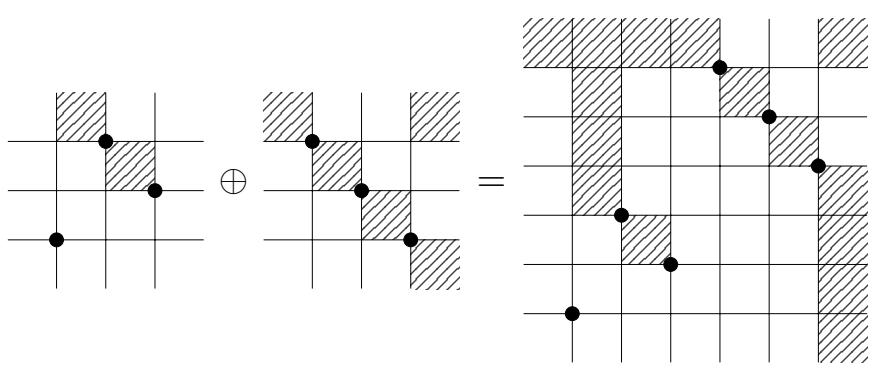

Figure 5.1: The direct sum of two mesh patterns.

or $\left(\left|s_{c l}\right|, j\right)$, shaded all the boxes north, east, south or west of the box, respectively, for all $0 \leq i<\left|s_{c l}\right|$ and $\left|s_{c l}\right|<j \leq\left|s_{c l}\right|+\left|t_{c l}\right|$. We similarly define the skew-sum for when the bottom right corner of $s$ and top left corner of $t$ are not shaded.

The direct product $s \oplus t$ can be considered as placing a copy of $t$ north east of $s$ and any shaded box that was on a boundary we extend to the new boundary, see Figure 5.1. We define the direct sum in this way because it maintains one of the most important properties in the permutation sense, that the first $\left|s_{c l}\right|$ letters are an occurrence of $s$ and the final $\left|t_{c l}\right|$ letters are an occurrence of $t$.

A permutation is said to be indecomposable if it cannot be written as the direct sum of smaller permutations. We generalise this to mesh patterns.

Definition 5.2. A mesh pattern $m$ is indecomposable (resp. skewindecomposable) if it cannot be written $m=a \oplus b$ (resp. $m=a \ominus b$ ), where neither a nor $b$ is $m$.

Remark 5.3. It is well known that a permutation has a unique decomposition into indecomposable permutations. This implies that a mesh pattern also has a unique decomposition.

Using these definitions we can give a large class of strongly disconnected intervals, which is a mesh pattern generalisation of Lemma 4.2 in [MS15].

Lemma 5.4. If $m$ is indecomposable, $h(m)>1$ and $(0,0),(|m|,|m|) \notin m_{\text {sh }}$, then $[m, m \oplus m]$ is strongly disconnected.

Proof. By Lemma 4.2 in [MS15] the interval $\left[m_{c l}, m_{c l} \oplus m_{c l}\right]$ is strongly disconnected, with components $P_{1}=\left\{m_{c l} \oplus x \mid x \in\left[1, m_{c l}\right)\right\}$ and $P_{2}=$ $\left\{x \oplus m_{c l} \mid x \in\left[1, m_{c l}\right)\right\}$. Consider any pair $\alpha, \beta \in[m, m \oplus m]$, if $\alpha_{c l}$ and $\beta_{c l}$ 
are not in the same component of $\left[m_{c l}, m_{c l} \oplus m_{c l}\right]$, then $\alpha$ and $\beta$ are incomparable. Let $\hat{P}_{1}=\left\{\alpha \mid \alpha_{c l} \in P_{1}\right\}$ and $\hat{P}_{2}=\left\{\alpha \mid \alpha_{c l} \in P_{2}\right\}$. However, $\hat{P}_{1} \cup \hat{P}_{2} \neq(m, m \oplus m)$ because it does not include the mesh patterns $\alpha$ with $\alpha_{c l}=m_{c l} \oplus m_{c l}$.

There are exactly two occurrences of $m$ in $m \oplus m$. These are $\eta_{1}$ the first $|m|$ letters and $\eta_{2}$ the last $|m|$ letters. Note that each shaded box of $m \oplus m$ is used by at least one of $\eta_{1}$ and $\eta_{2}$, so if we deshade a box the resulting pattern $x$ contains at most one occurrence of $m$, either the first or last $|m|$ letters. Let $Q_{1}$ and $Q_{2}$ be sets of patterns with underlying permutation $m_{c l} \oplus m_{c l}$ where the first and last $|m|$ letters are the only occurrence of $m$, respectively. So any element $Q_{1}$ cannot contain an element in $P_{2} \cup Q_{2}$ and similarly any element of $Q_{2}$ cannot contain an element of $P_{1} \cup Q_{1}$. Finally, note that $P_{1} \cup Q_{1}$ is a connected component as it has the minimal element $m_{c l} \oplus 1$, similarly $P_{2} \cup Q_{2}$ is connected as it has the minimal element $1 \oplus m_{c l}$. Therefore, $P_{1} \cup Q_{1}$ and $P_{2} \cup Q_{2}$ are disconnected nontrivial components of $[m, m \oplus m]$.

Corollary 5.5. If $m$ is skew-indecomposable, $(|m|, 0),(0,|m|) \notin m_{\text {sh }}$ and $h(m)>1$, then $[m, m \ominus m]$ is strongly disconnected.

Using Lemma 4.2 in [MS15] it is shown that almost all intervals of the classical permutation poset are not shellable. The proof of this follows from the Marcus-Tardos theorem. We have seen this result does not apply in the mesh pattern case, so we cannot prove a similar result using this technique. A similar problem was studied for boxed mesh patterns in permutations in [AKV13], which is equivalent to boxed mesh patterns in fully shaded mesh patterns. So we present the following open question:

Question 5.6. What proportion of intervals of $\mathcal{M}$ are shellable?

The Möbius function in the permutation poset can be computed more easily by decomposing the permutations into smaller parts using the direct sum, or skew-sum, see [BJJS11, MS15]. Which leads to the following question:

Question 5.7. Can a formula for the Möbius function of $\mathcal{M}$ be obtained by decomposing mesh patterns using direct sums and skew sums?

\section{Acknowledgements}

We would like to thank the anonymous referee for their extremely useful comments and corrections which greatly improved the paper. 
[AKV13] Sergey Avgustinovich, Sergey Kitaev, and Alexandr Valyuzhenich. Avoidance of boxed mesh patterns on permutations. Discrete Applied Mathematics, 161(12):43 - 51, 2013.

[BC11] Petter Brändén and Anders Claesson. Mesh patterns and the expansion of permutation statistics as sums of permutation patterns. Electron. J. Combin, 18(2):P5, 2011.

[BJJS11] Alexander Burstein, Vít Jelínek, Eva Jelínková, and Einar Steingrímsson. The Möbius function of separable and decomposable permutations. Journal of Combinatorial Theory. Series A, 118(8):2346-2364, 2011.

[Bjö90] Anders Björner. The Möbius function of subword order. Institute for Mathematics and its Applications, 19:118, 1990.

[CTU15] Anders Claesson, Bridget Eileen Tenner, and Henning Ulfarsson. Coincidence among families of mesh patterns. The Australasian Journal of Combinatorics, 63:88-106, 2015.

[Hat02] Allen Hatcher. Algebraic Topology. Cambridge University Press, 2002.

[JKR15] Miles Jones, Sergey Kitaev, and Jeffrey Remmel. Frame patterns in n-cycles. Discrete Mathematics, 338(7):1197-1215, July 2015.

[MS15] Peter R. W. McNamara and Einar Steingrímsson. On the topology of the permutation pattern poset. Journal of Combinatorial Theory, Series A, 134:1-35, 2015.

[MT04] Adam Marcus and Gábor Tardos. Excluded permutation matrices and the Stanley-Wilf conjecture. Journal of Combinatorial Theory, Series A, 107(1):153-160, 2004.

[Qui78] Daniel Quillen. Homotopy properties of the poset of nontrivial p-subgroups of a group. Advances in Mathematics, 28(2):101-128, 1978.

[Smi16] Jason P. Smith. Intervals of permutations with a fixed number of descents are shellable. Discrete Mathematics, 339(1):118 - 126, 2016.

[Smi17] Jason P. Smith. A formula for the Möbius function of the permutation poset based on a topological decomposition. Advances in Applied Mathematics, 91:98 - 114, 2017. 
[SV06] Bruce E. Sagan and Vincent Vatter. The Möbius function of a composition poset. Journal of Algebraic Combinatorics, 24(2):117-136, 2006.

[TU18] Murray Tannock and Henning Ulfarsson. Equivalence classes of mesh patterns with a dominating pattern. Discrete Mathematics and Theoretical Computer Science, Special issue for Permutation Patterns 2016, vol. 19, 2018.

[Wac07] Michelle L. Wachs. Poset topology: Tools and applications. In Geometric Combinatorics, volume 13 of IAS/Park City Math. Ser., pages 497-615. Amer. Math. Soc., 2007. 